\title{
Multiple frequencies multiple carriers
}

\author{
Myungsup Kim*, and Do Young Kwak \\ Department of Mathematical Sciences, Korea Advanced Institute of Science and Technology, 291, \\ Daehak-ro, Yusung-gu, Daejeon, Republic of Korea, 34141
}

\begin{abstract}
The OFDM scheme has the problems of out-of-band (OOB) leakage and low transmission efficiency in the time domain. This is because the scheme is a single frequency single carrier one in which one carrier is generated by one frequency. To overcome the problems we propose multiple frequencies multiple carriers (MFMC) scheme in which one carrier is generated using many frequencies. We establish a practical model which not only eliminates these disadvantages but also improves the transmission efficiency and peak-to-average power ratio (PAPR) performance. In the proposed MFMC scheme, the filter matrix is used in order to confine the OOB leakage of the spectrums of transmit signal, which is generated through processes such as jump-removing, filtering, and orthogonalizing from an initial matrix. MFMS is evaluated compared with OFDM in view of transmission efficiency, OOB leakage, and bit error rate performance.
\end{abstract}

\section{Introduction}

Since the introduction of OFDM, it has been used as a transmission technology in many fields because of the efficient use of frequency [1]. However, an OFDM transmission system in which a single carrier has a single frequency is not easy to use as a future technology until the OOB leakage problem is solved. In order to solve this problem, many people have developed orthogonality and frequency-efficient techniques between carriers using precoding technology [2-4]. These techniques attempt to solve the OOB power leakage problem of OFDM by replacing the conventional square wave with smooth envelopes. Recently, there has been an attempt to improve the efficiency of spectrum by using nonorthogonal functions among subbands. Typically, there are universal filtered multi-carrier (UFMC) and generalized frequency division multiplexing (GFDM) [5, 6]. Since the envelope function of OFDM is in the form of a square wave, Kim and Kwak proposed methods of starting the symbol from 0 to improve the disadvantage that the amplitude does not disappear rapidly from the center [7,8]. They also tried to apply the method to the mobile communication [9]. However, since the PAPR problem and the BER performance are lacking in detail, it is pointed out that there still needs to be studied for practical application.

In this paper, we propose a scheme whose careers are created by using multiple frequencies; it can confine the occupied band and maintain the orthogonality among the

\footnotetext{
* Corresponding author: myungsup@kaist.ac.kr
} 
careers, so that it can exert the maximum BER performance because carriers are orthogonal each other. We will use diagonal matrices as an initial matrix to maintain orthogonality while confining the bandwidth of careers.

In transmission technology, the efficiency in the frequency domain is important, but the efficiency in the time domain is also important. For example, when the FFT size is 1024 in LTE (Long Term Evolution) specification [10], the number of occupied carriers is 600 . Because the channel bandwidth is $10 \mathrm{MHz}$ and the used frequency band is $9 \mathrm{MHz}$, it has $90 \%$ efficiency in the frequency domain. However, as seen from the time domain, since the number of symbols transmitted in 1024 samples is 600 , the transmission efficiency is $58.6 \%$. Therefore, this should be improved. In this paper, we aim to improve the usage efficiency to more than $90 \%$ in the time domain.

\section{MFMC}

\subsection{System description}

The MFMC system has orthogonality in which carriers share the entire band within a given band. Since the subcarriers used in the MFMC system are composed of a sum of several frequencies, they can be expressed in a limited band. Therefore, the OOB leakage problem can be solved and the length of the symbol can be minimized. Figure 1 shows the transmitter of the MFMC system. Matrix C block and DFT block are placed in front of the matrix to improve PAPR. The filter matrix $G$ serves to limit the band of the input data symbol, and the signal is transformed into the time domain from the frequency domain through IFFT.

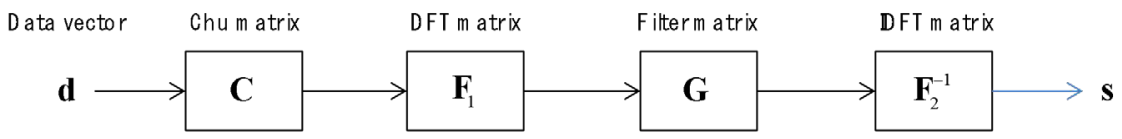

Fig. 1. The transmitter in the MFMC communication system.

We will use the expression MFMC ( , ) where and are the FFT size and the number of subcarriers, respectively. The transmitted signal can be expressed as:

$$
\begin{aligned}
\mathbf{s} & =\mathbf{F}_{2}^{-1} \mathbf{G F}_{1} \mathbf{C d} \\
& =\mathbf{U d}
\end{aligned}
$$

where $\mathbf{S}$ is the transmit signal vector $\left[s_{0}, s_{1}, \cdots, s_{L-1}\right]^{T}, \mathbf{d}$ is the input data vector $\left[d_{0}, d_{1}, \cdots, d_{M-1}\right]^{T}, \mathbf{C}$ is the Chu matrix $\operatorname{diag}\left(e^{j n^{2} / M} \mid n=0,1, \cdots, M-1\right), \mathbf{F}_{1}$ is the DFT matrix $\left[f_{m, n}\right]_{\substack{m=0, \ldots M-1, n=0, \ldots, M-1,}}$ where $f_{m, n}=e^{-j \frac{2 \pi m n}{N}}, \mathbf{G}$ is the filter matrix $\left[g_{m, n}\right]_{\substack{m=0, \ldots, L-1, n=0, \ldots, M-1}}, \mathbf{F}_{2}$ is the FFT matrix $\left[f_{m, n}\right]_{\substack{m=0, \ldots L-1,1 \\ n=0, \ldots, L-1}}$, and $\mathbf{U}$ is the transfer matrix $\left[u_{m, n}\right]_{\substack{m=0, \ldots, L-1, n=0, \ldots, M-1 .}}$

\subsection{Generation of the filter matrix, $G$}

The filter matrix is generated by the following expression

$$
\mathbf{G}=f\left(\mathbf{F}_{2} \mathbf{\Phi} \mathbf{F}_{2}^{-1} \mathbf{G}_{0}\right),
$$

where 


$$
f(\mathbf{K})=\mathbf{K}\left(\mathbf{K}^{H} \mathbf{K}\right)^{-\frac{1}{2}}
$$

Figure 2 shows the process of generating the filter matrix.

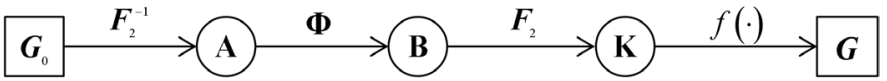

Fig. 2. Filter matrix generation flow diagram.

\subsection{Initial Matrix}

There may be a myriad of initial matrices for generating filter matrices. However, in this paper, the simplest initial matrix is defined as follows.

$$
\mathbf{G}_{0}=\left(\begin{array}{cc}
\mathbf{I}_{\frac{M}{2} \times \frac{M}{2}} & \mathbf{0}_{\left(L-\frac{M}{2}\right) \times \frac{M}{2}} \\
\mathbf{0}_{\left(L-\frac{M}{2}\right) \times \frac{M}{2}} & \mathbf{I}_{\frac{M}{2} \times \frac{M}{2}}
\end{array}\right),
$$

where $L$ is the FFT size and $M$ is the length of any data vector. That is an initial matrix whose columns represent frequency components and whose rows represent channels for transmitting $M$ data symbols. In this initial matrix, each subchannel has only one frequency component, because each column contains one element having a value of 1 among all elements and the remaining elements have a value of zero.

IFFT for each column can be expressed as follows.

$$
a(p)= \begin{cases}\frac{1}{L} e^{j \frac{2 \pi p m}{L}}, \quad p \leq \frac{M}{2}, \\ \frac{1}{L} e^{j \frac{2 \pi(L-p) m}{L}}, \quad p>L-\frac{M}{2} .\end{cases}
$$

After the convolution with the filter, we make the length of the symbol equal to the original length at the beginning and the end of the sample sequence $\{a(k)\}$. For example, $I$ samples are removed before and after the sample sequence $\{a(k)\}$ when the filter length is $2 I+1$. After doing so, the first sample in the truncated sequence is set to zero. Then, (5) becomes as follows.

$$
\bar{a}(p)=\left\{\begin{array}{l}
\left\{\begin{array}{c}
0, \quad m=0, \\
\frac{1}{L} e^{j \frac{j \pi p m}{L}}, 1 \leq m \leq L-2 I,
\end{array} \quad p \leq \frac{M}{2},\right. \\
\left\{\begin{array}{c}
0, \quad m=0, \\
\frac{1}{L} e^{j \frac{2 \pi(L-p) m}{L}}, 1 \leq m \leq L-2 I,
\end{array} \quad L-\frac{M}{2}<p \leq M .\right.
\end{array}\right.
$$

The FFT with a length of $L$ is obtained as follows.

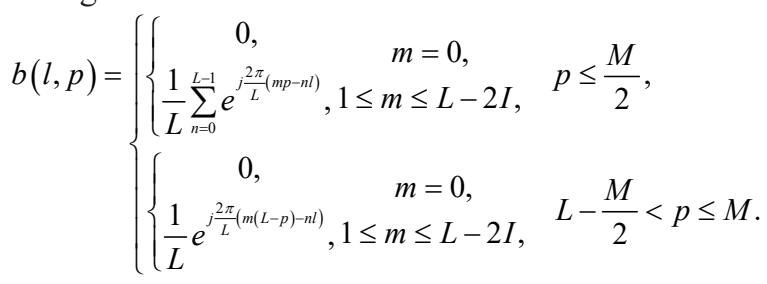

It can be seen that the frequency components of the $p$-th subchannel are synthesized with $L$ frequencies. $b(l, p)$ is the $(l, p)$-th entry of the matrix B. 


\subsection{Filtering}

The filtering is performed by the following matrix.

$$
\boldsymbol{\Phi}=\frac{1}{4}\left[\begin{array}{cccccc}
1 & & & & & \\
2 & 1 & & & & \\
1 & 2 & 1 & & & \\
& & \ddots & & & \\
& & & 1 & 2 & 1 \\
& & & & 1 & 2 \\
& & & & & 1
\end{array}\right] .
$$

\subsection{Nearest Orthogonal Matrix}

The matrix $\mathrm{K}$ is generated through jump removing and internal filtering using $\boldsymbol{\Phi}$ in the time domain, or $\boldsymbol{\Omega}$ in the frequency domain. However, even if an initial matrix $\mathbf{G}_{0}$ is orthogonal, the matrix $\mathrm{K}$ can be no longer guaranteed to be orthogonal. Therefore, the matrix $\mathrm{K}$ should be transformed to be orthogonal while retaining its original properties as near as possible. We can obtain a filter matrix $\mathrm{G}$ which is nearest to $\mathrm{K}$, whose columns are orthogonal to each other through the following formula [11].

$$
\mathbf{G}=f(\mathbf{K})=\mathbf{K}\left(\mathbf{K}^{H} \mathbf{K}\right)^{-\frac{1}{2}} .
$$

\subsection{Illustration with MFMC $(128,116)$}

The transmission efficiency is improved by making the FFT size and the length of the data vector similar. The MFMC $(128,116)$ scheme uses 116 out of 128 careers, so they use $90.6 \%$ of careers out of all careers. Such a scheme achieves the same efficiency as 116 samples for 128 samples transmitted in the time domain. Figure 3(a) shows the response characteristics in the frequency domain of OFDM. Because the magnitude of the signal at both ends of the frequency is large, it can invade neighbouring channels. Figure 3(b) shows the response characteristics in the frequency domain of the MFMC scheme. Since the magnitude of the carrier is blocked at both ends of the frequency, it can be seen that the neighbouring channel cannot be invaded.

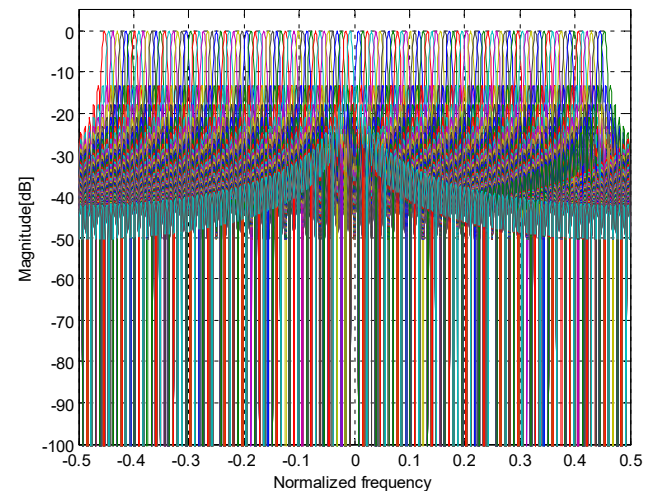

(a)

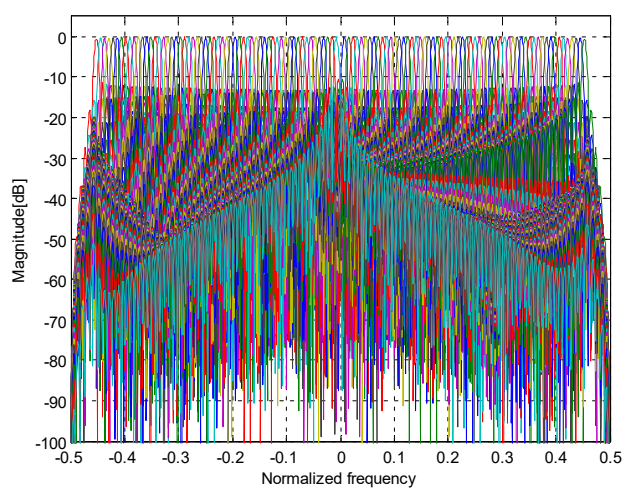

(b)

Fig. 3. (a) Normalized frequency response of OFDM, (b) Normalized frequency response of $\operatorname{MFMC}(128,116)$. 


\section{BER performance}

Since the performance related matrix is $\mathbf{G}$ in the transmitter in figure 1, we have the BER performance of MFMC with QPSK data symbols as

$$
p_{b}=\frac{1}{2} \operatorname{erfc}\left(\sqrt{\frac{E_{b}}{N_{o}} \frac{\operatorname{tr}\left(\mathbf{G}^{H} \mathbf{G G}^{H} \mathbf{G}\right)}{\operatorname{tr}\left(\mathbf{G G}^{H}\right)}}\right)
$$

where $E_{b}$ is the energy per bit and $N_{o} / 2$ is the noise power spectral density. Figure 4 shows the BER performance of the $\operatorname{MFMC}(128,116)$. It is shown that the theoretic and simulation results agree with well. It is because orthogonality between the careers is maintained over additive white Gaussian noise (AWGN) channel, so they do not interfere with each other.

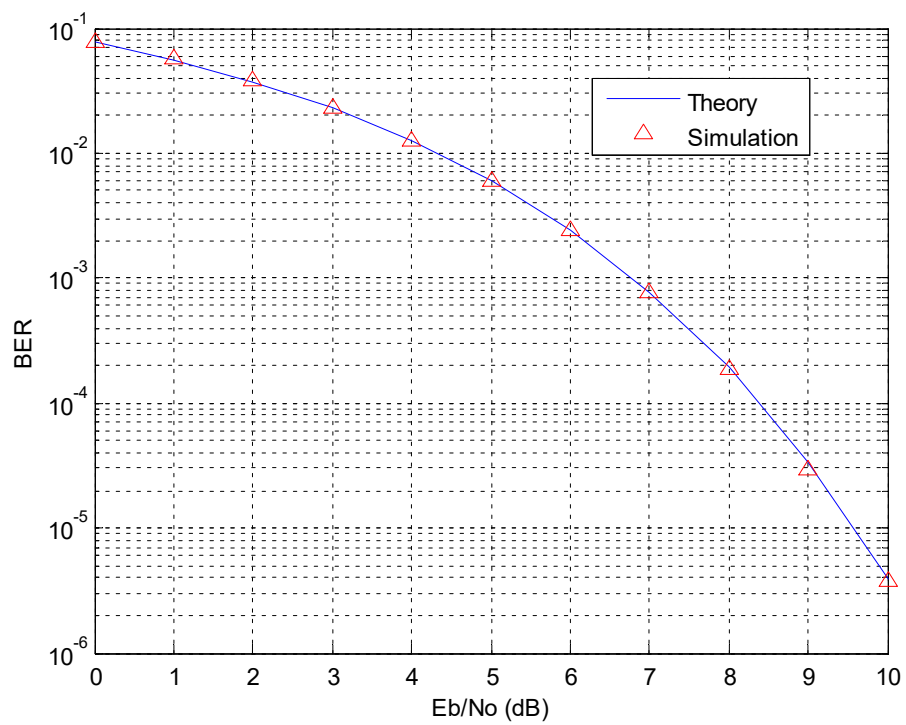

Fig. 4. BER performance of MFMC with QPSK.

\section{Conclusion}

In order to solve the OOB leakage problem of OFDM which has an intrinsic problem of the conventional SFSC scheme, we proposed a MFMC scheme which had the bandwidth confine, the OOB leakage protection, and the similar CCDF performance with DFT-OFDM. It was also confirmed that orthogonality between carriers was guaranteed because the BER performance of the MFMC agreed with exactly theoretical one. In addition, since the performance was obtained under the condition that occupied careers are $90 \%$ of the entire carriers, it was verified that the transmission efficiency could be increased not only in the frequency domain but also in the time domain.

It is expected that the proposed MFMC method will be a theoretical basis to create new carriers which have various functions with frequency band confine, transmission efficiency in the time domain, spectral efficiency, and orthogonality simultaneously for future wireless communications.

\section{References}


1. Stephen B. Weinstein, "The History of Orthogonal Frequency-Division Multiplexing," IEEE Communications Magazine, vol. 47, no. 11, pp. 26-35, Nov. (2009).

2. Char-Dir Chung and Kuo-Wei Chen, "Spectrally Precoded OFDM Without Guard Insertion," IEEE Trans. VEHICULAR TECHNOLOGY, VOL. 66, NO. 1, pp. 107-121, Jan. (2017).

3. Char-Dir Chung, "Spectrally Precoded OFDM," IEEE Trans. Comm., VOL. 54, NO. 12, pp. 2173-2185, Dec. (2006).

4. Jaap van de Beek, "Orthogonal Multiplexing in a Subspace of Frequency WellLocalized Signals,” IEEE Comm. LETTERS, VOL. 14, NO. 10, pp. 882-884, OCTOBER (2010).

5. F. Schaich, T. Wild, "Waveform contenders for 5G - OFDM vs. FBMC vs. UFMC", Proceedings of 6th International Symposium on Communications, Control, and Signal Processing (ISCCSP 2014), Athens, Greece, May (2014).

6. Nicola Michailow, Maximilian Matthé, Ivan Simões Gaspar, Ainoa Navarro Caldevilla, Luciano Leonel Mendes, Andreas Festag, and Gerhard Fettweis, "Generalized Frequency Division Multiplexing for 5th Generation Cellular Networks," IEEE Trans. Comm. VOL. 62, NO. 9, pp 3045 - 3061, Sep. (2014).

7. Myungsup Kim and Do Young Kwak, "COLUMN MEAN VANISHING MATRICES," International Journal of Pure and Applied Mathematics, Vol. 114, No. 3, pp. 457-471, (2017).

8. Myungsup Kim and Do Young Kwak, "A PILOT INCLUDED COLUMN MEAN VANISHING MATRIX," Journal of Mathematics Research, Vol. 9, No. 2, pp 128 133, April (2017).

9. Myungsup Kim and Do Young Kwak, 'Generalized OFDM for $5^{\text {th }}$ Generation Mobile Communication,' Accepted Paper, VTC2017-Spring, (2017).

10. LTE; Evolved Universal Terrestrial Radio Access (E-UTRA); User Equipment (UE) radio transmission and reception (3GPP TS 36.101 version 8.15.0 Release 8)

11. Nicholas J. Higham, "Computing the Polar Decomposition - With Applications*," SIAM J. SCI. STAT. COMPUT. Vol. 7, No. 4, October (1986). 